\title{
INSTRUMENTATION \& TECHNIQUES A precision tooth-pulp stimulation technique for the assessment of pain threshold
}

\author{
BARRY R. DWORKIN \\ Rockefeller University, New York, New York 10021
}

and

\author{
MATHEW H. M. LEE, HERBERT H. ZARETSKY, and HOPE A. BERKELEY \\ Department of Rehabilitation Medicine, Goldwater Memorial Hospital, \\ New York University Medical Center, Roosevelt Island, New York 10044
}

\begin{abstract}
Accurate and consistent pain threshold measurements for the tooth pulp can be achieved with careful preparation of a moisture-proof appliance and the use of a high-quality constantcurrent stimulator. Using an extant dental filling as the cathode, the usual problems associated with the control of the stimulation area and locus and, consequently, current density can be overcome. Repeated measurements showing less than $\pm 5 \%$ variation over many months can be routinely achieved on the same individual. The technique has shown a high degree of sensitivity to common analgesic agents and is potentially useful for the investigation of variations in pain threshold over extended periods of time.
\end{abstract}

The reliable placement of a precisely defined stimulus is the primary requirement for refined psychophysical measurement. For accurate, parsimonious scaling with maximum experimental generality, stimulation techniques that produce qualitatively consistent sensation over a wide dynamic range are especially desirable. The electrical stimulation of dental fillings for the assessment of changes of pain sensitivity in humans can meet these criteria if adequate attention is given to technical detail.

In our laboratory, repeated threshold measurements varying less than $\pm 5 \%$ over many months can be routinely achieved on the same individual. High sensitivity to common analgesic agents and systematic variation with psychological and physiological manipulations are readily demonstrated. Since virtually any detectable level of dental nerve stimulation is reported as distinctly unpleasant, the complex succession of stimulus qualities normally associated with pressure, heat, or other types of electrical stimulation is absent, and detection threshold data correlate very well with higher levels of discomfort. Consequently, most experiments can be confined to levels of stimulation that are both completely safe and humane. The sensation associated with electrical stimulation of dental fillings is reported by all subjects to cease almost simultaneously with termination of the current, and the energy levels applied are well below those which would be suspected of producing tissue damage. Electrical dental-filling stimulation closely approximates a kind of pain which is of clinical

The authors gratefully acknowledge the advice and assistance of Dr. Lawrence Eisenberg. importance, with which most individuals have had at least some previous experience, and for which common analgesic agents are frequently prescribed.

With the advent of good constant-current stimulators, the major problem in pain threshold measurement has been consistent electrode geometry relative to the receptive field. A dental filling and the innervation of the associated pulp are embedded in a rigid saline-filled bony matrix; consequently, the geometry of the conduction path is relatively invariant. The current density through any region of the nerve is a fixed fraction of the net current flowing from the electrode (the filling) to ground (the gum). Thus, to assure precise and repeatable stimulation, two requirements must be met: (1) The current must be generated accurately, and (2) all of the current generated must flow through the filling to ground and not over the surface of the tooth. The enamel of the tooth is a relatively good insulator when dry. The enamel must be kept dry and good contact must be made to the surface of the filling.

A variety of other investigators have attempted to use dental pulp stimulation (Andersson, Ericson, Holmgren, \& Lindquist, 1973; Chatrian, Canfield, Knauss, \& Lettich, 1975; Savara, Fields, \& Tacke, Note 1). For one reason or another, we have found their techniques to be less satisfactory for our purposes than the procedure reported here. However, it is fair to say that our method is quite time consuming. It was designed to optimize accuracy, reliability, and comfort for the subject with little concern for convenience or expense. Once a subject has been correctly prepared, threshold readings can ordinarily be taken over many hours without additional attention to the stimulation apparatus. 


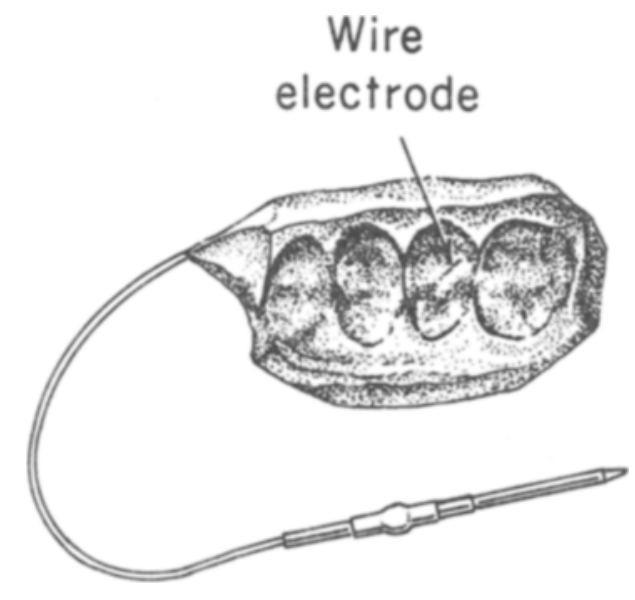

Figure 1. Silicone rubber mold showing electrode and Tefloninsulated connecting wire.

\section{PREPARATION OF THE ELECTRODE ASSEMBLY}

Stimulation is delivered to the surface of the dental filling by means of a tightly fitting moisture-proof electrode assembly, individually prepared for each subject. This flexible dental mold is constructed of commercially available silicone rubber and requires several steps for preparation (Figure 1).

First, a suitable filling is selected for use as the stimulation site. The criteria for such a filling are: (1) The dental nerve innervating the tooth is intact; (2) the amalgam has been in place for at least 1 year, so that shifting of the material is minimized; and (3) the filling is physically isolated from the gingiva and adjacent fillings to avoid current leakage.

Next, a partial impression is taken of the desired area using a "tray-type" silicone impression material such as Pfingst Lastic 55 silicone elastic impression material or Sterndent SIR. ${ }^{1}$ This impression is poured with fine dental plaster to produce a positive template. The stimulating electrode (Grass E-2B EEG platinum needle) is implanted tip down approximately $1 / 4 \mathrm{in}$. in the plaster model at the site of stimulation. This allows for accurate positioning of the electrode tip when the silicone rubber mold is cast.

General Electric RTV 631 two-package silicone rubber is used for the final electrode assembly. The uncured rubber base is mixed with catalyst and aspirated in a partial vacuum according to manufacturer's instructions. Then it is poured into a shallow container so that the plaster/electrode template, which has been treated with Vaseline as a separating medium, can be embedded in the mixture. Curing is completed with $1 / 2 \mathrm{~h}$ at $100^{\circ} \mathrm{C}$. When cool, the plaster is removed, leaving the stimulating electrode protruding $1 / 4$ in. from the rubber mold just above the selected filling. The mold can be trimmed to maximize comfort so that it includes only three or four teeth surrounding the stimulation site. Finally, to produce a "spring-like" effect when the preparation is in use, the electrode tip is bent at a $45-\mathrm{deg}$ angle with a pair of small pliers.

\section{DELIVERY OF STIMULATION}

At the start of each experimental session, the rubber mold is carefully affixed to the stimulation site, where it remains for the duration of the period. The filling to be stimulated and the surrounding area are thoroughly dried with cotton rolls, compressed air, and a dental drying agent such as Prep Dry. The mold is prepared with a thin coating of Vaseline and filled with Sterndent SIR impression material. It is then inserted and held in place until the impression material, which acts as a liquid-proof seal, has solidified.

An indifferent electrode is constructed of a solid gold bar $5 \times 10 \times 2 \mathrm{~mm}$, drilled along the greater axis with a 5-mm-deep, 1-mm-diam hole into which the stripped portion of a Teflon-insulated wire is crimped. Gold is necessary to avoid electrolytically produced sensations of taste, and care must be taken to have at least $3 \mathrm{~mm}$ of Teflon insulation inside the bar. If the edges and corners of the bar are carefully rounded, it will fit comfortably into the buccal pouch adjacent to the filling to be stimulated. The bar is connected to the anode of the stimulator and the filling to the cathode. The surface area of the bar is sufficient that the current density at the gum surface remains below threshold.

Figure 2 shows a block diagram of the stimulator circuit. The logic which generates the pulse pattern is operated from the mains. However, the stimulator current source operates from a battery and is optically isolated from the logic. The stimulation current is monitored by measuring the voltage across a $1-\mathrm{K} \Omega$ resistor in series with the preparation. The amplifier connected to the resistor is a medical instrumentation unit which employs high-frequency transformer

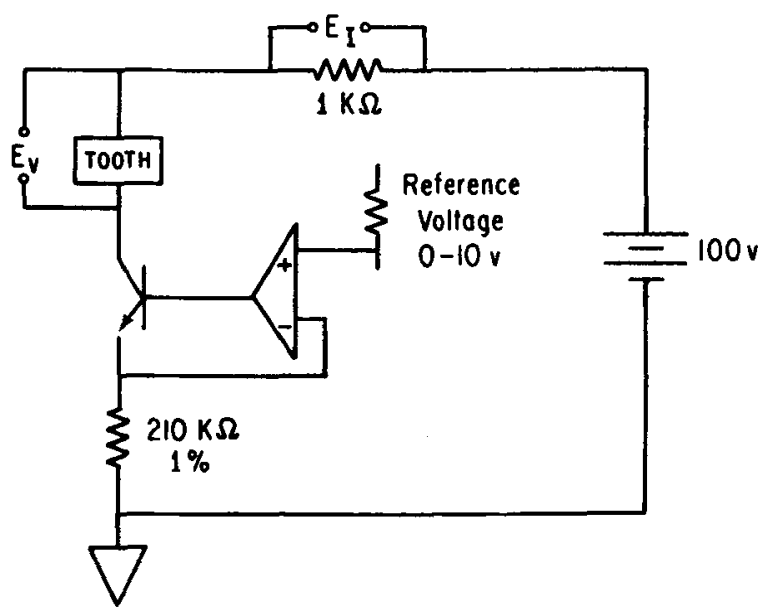

Figure 2. A partial schematic diagram of the current control section of the stimulator. Note that the reference ground is isolated. $E_{1}$ and $E_{y}$ are used to calculate the preparation impedance. 


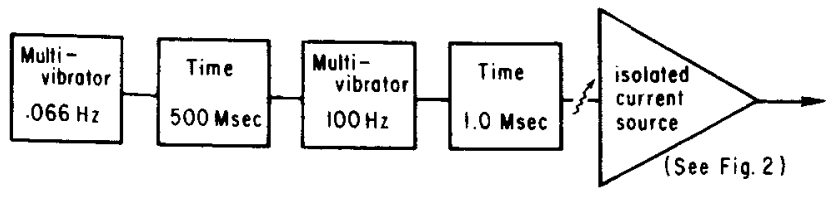

Figure 3. A block diagram of the stimulator system.

coupling of the input stage and its power supply to achieve electrical isolation. The output voltage of the stimulator is monitored with a similar amplifier. The signals from these amplifiers representing the stimulus pulse voltage and current are displayed simultaneously on a two-channel storage oscilloscope. From the display, calculation of the preparation impedance is simple. Impedance cannot be measured with a standard highsensitivity ohmmeter because the interface between the filling and the underlying tissue frequently generates an electrochemical potential which either adds or subtracts from the current source in the instrument, producing an erroneous reading. It is important to monitor the impedance, particularly throughout long sessions, to detect problems with the preparation which could affect the results.

These problems may be of two different types: (1) Very large increases in impedance may be caused by poor contact between the platinum electrode and the filling. However, since the compliance of the stimulator is greater than $100 \mathrm{~V}$, this problem is unusual and the impedance must become very large before it alters the current passing through the filling. At a stimulation level of 100 microA, the net preparation impedance must exceed $1 \mathrm{M} \Omega$ before the compliance fails. However, large increases $(>10 \%)$ in impedance during a measurement session suggest poor technique in either placing or constructing the appliance and should be investigated. (2) Decreased impedance almost always signals that saliva has leaked into the space between the appliance and the tooth. This condition must be corrected immediately by removing, drying, and reinserting the appliance because it is not compensated for by the stimulator circuit. Inaccurately high thresholds are obtained under this circumstance because, by the law of parallel resistances, the stimulation current will be proportionately divided between the "sneak path" through the saliva and the effective path through the filling and the nerve.

A partial block diagram of the stimulator is shown in Figure 3. The logic for generating the stimulation pulse trains is conventional. The stimulator current control section is isolated from the logic by an L.E.D./photodiode pair. The current control is a grounded base arrangement (see Figure 2) in which the emitter current is constrained to the selected value by an operational amplifier. Since $I_{\mathfrak{c}}=\alpha I_{\mathfrak{e}}$, the current flowing in the collector circuit, of which the preparation is a series element, is maintained constant independently of large variations in preparation impedance. Any good isolated stimulator can be used. The general design we have chosen provides high precision and a wide compliance over the dynamic range of 0.500 microA. It also is efficient, because maximum voltage of the device is fully available at the preparation. The importance of safe conservative design cannot be over-stressed. There is no danger to the subject if the stimulation current is confined to the path between the tooth and the indifferent electrode in the adjacent gum area.

In our standard procedure, stimulation is delivered every $15 \mathrm{sec}$ as a train of 500 -msec duration consisting of $501 \mathrm{-msec}$ pulses (Figure 3). Intensity of the stimulus, as determined by the current passing through the circuit, can be gradually increased or decreased by the experimenter within the stimulator range of $0-500$ microA. The subject signals his or her perception of the stimulus by pressing a button which lights the experimenter's panel.

Measurements are made at two perceptual levels, detection and discomfort. For the detection threshold trials, the subject is instructed to respond each time he or she notices the presence of the stimulus. During the first session, threshold determinations are made, using uniform current increments. Once the vicinity of the threshold has been established, determinations begin at $80 \%$ of the previous threshold value and the current is increased by $2 \%$ until the subject responds three times at the same level. The average value of two determinations is recorded as the detection threshold. For discomfort threshold measurement, the subject receives the following instructions: "This time, press the button only when the stimulus is clearly uncomfortable. We are not interested in how much pain you can tolerate but, rather, in the point at which the stimulus crosses the pain threshold. If you like, think to yourself, 'Would I take aspirin if this sensation persisted?' We would like you to press the button only when the sensation reaches a level of discomfort that would prompt you to take aspirin." Stimulation is gradually increased by the same procedure as was used to determine the detection threshold until a level is reached at which the subject responds three times consecutively.

\section{REFERENCE NOTES}

1. Savara, B. S., Fields, R. W., \& Tacke, R. B. Application of electrical current in dental anesthesia. Annual summary report of the Dental Research Branch, U. S. Army Medical Research and Development Command. Washington, D. C., 1974.

\section{REFERENCES}

Andersson, S. A., Ericson, T.. Holmgren, E., \& Lindovist, G. Electroacupuncture: Effect on pain threshold measured with electrical stimulation of teeth. Brain Research, 1973, 63. 393-396.

Chatrain, G. E., Canfield, R. C., Knauss, T. A., \& Lettich, E. Cerebral responses to electrical tooth pulp stimulation in man. Neurology. 1975, 25, 745-757.

\section{NOTES}

1. Pfingst Inc., 62 Cooper Square, New York, New York 10003; Weber Consumable Products, Division of Sterndent Corporation, Mt. Vernon, New York 10553.

(Received for publication April 7, 1977; revision accepted June 7,1977 .) 